\title{
Torus Embedded Hypercube Interconnection Network: A Comparative Study
}

\author{
N. Gopalakrishna Kini \\ Department of CSE \\ Manipal Institute of Technology (Manipal \\ Universitv). Manipal, INDIA 09448931570
}

\author{
M. Sathish Kumar \\ School of EECS, \\ Seoul National University, Seoul, SOUTH \\ KOREA
}

\author{
Mruthyunjaya H.S. \\ Department of $E$ \& $C$ \\ Manipal Institute of Technology (Manipal \\ University), Manipal, INDIA
}

\begin{abstract}
A design analysis and comparison of a product network generated from torus and hypercube networks known as torus embedded hypercube scalable interconnection network suitable for parallel computers is presented in this paper. It is shown here that with minor modifications in architecture of the existing mesh embedded hypercube interconnection network how good a torus embedded hypercube interconnection network could be. Also it has been proved with the computational results that the torus embedded hypercube interconnection network is highly scalable and more efficient in terms of communication.
\end{abstract}

\section{Categories and Subject Descriptors}

C.2.1 [Computer Systems Organization]: ComputerCommunication Networks - Network Architecture and Design

\section{General Terms}

Performance, Design

\section{Keywords}

Hypercube network, Torus network, Mesh embedded hypercube network, Scalability, Network parameters.

\section{INTRODUCTION}

The effectiveness of parallel computers is often determined by its communication network. The interconnection network is an important component of a parallel processing system. A good interconnection network should have less topological network cost and meanwhile keep the network diameter as shorter as possible [2].

The mesh is a network with constant node degree in its internal nodes where as torus network has constant node degree with all its nodes [1]-[3]. The advantages of torus network can be imposed on to the mesh embedded hypercube network [4], [5] to give rise to an embedded architecture [6]-[8] called torus embedded hypercube scalable interconnection network and hence an architectural enhancement of mesh embedded hypercube network. torus embedded hypercube interconnection network compared to the existing mesh embedded hypercube interconnection network.

\section{ARCHITECTURAL PROPERTIES}

Let $l \times m$ be the size of several concurrent torus networks and $N$ be the number of nodes connected in hypercube configuration in it. Several hypercube connections can be derived from such group of $N$ nodes within the torus configuration [9], [10] as shown in Figure 1. With this torus embedded hypercube network can be labeled as a network of size $(l, m, N)$.

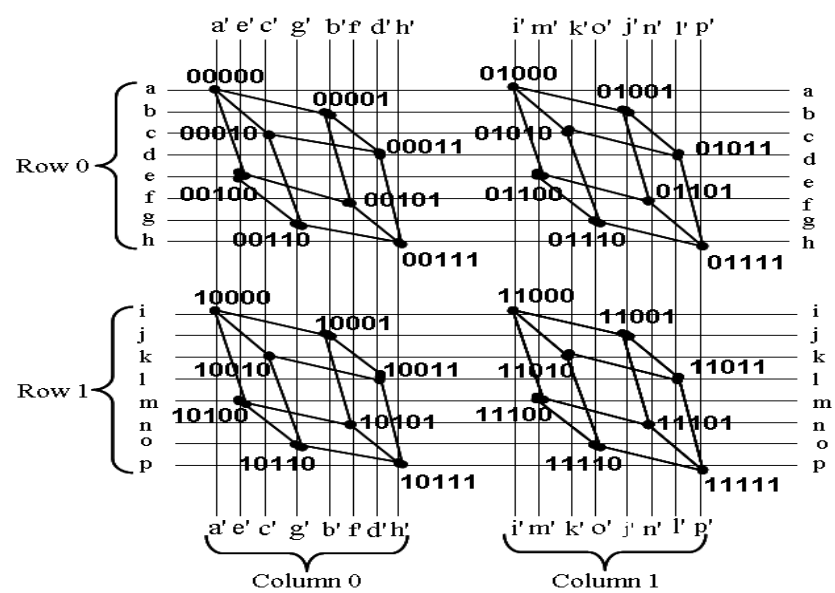

Figure 1. A $(2,2,8)$ - Torus embedded hypercube network

Each node in the network can be addressed with three components; row number $i$ and column number $j$ of torus appended with the address of node $k$ of hypercube. Hence, a $(l, m$, $N)$-torus embedded hypercube network will have $\quad l \times m \times N$ number of nodes and a node will be addressed as $(i, j, k)$ where 0 $\leq i<l, 0 \leq j<m$ and $0 \leq k<N$.

Combining the data routing functions of torus and hypercube networks to provide with the routing functions of the torus embedded hypercube [4] as

In this paper, we have described about the torus embedded hypercube network [9]. Also it has been proved how efficient a 
$T_{h_{1}}(i, j, k)=(i,(j+1) \bmod m, k)$

$T_{h_{2}}(i, j, k)=(i,(m+j-1) \bmod m, k)$

$T_{h_{3}}(i, j, k)=((i+1) \bmod l, j, k)$

$T_{h_{4}}(i, j, k)=((l+i-1) \bmod l, j, k)$

$T_{C_{d}}\left(k_{n-1} . k_{d+1} k_{d} k_{d-1} . . k_{0}\right)=\left(k_{n-1} \ldots k_{d+1} \overline{k_{d}} k_{d-1} \cdots k_{0}\right)$

We provide more explanation for the above discussion in the APPENDIX with a torus of size $2 \times 2$ and a 3 -cube hypercube and derive a $(2,2,8)$-torus embedded hypercube network by combining them as in Figure 1 and in (1) - (5).

In Figure1, the ring connections of row/column of each torus are not shown for simplicity and without that the network will be a (2, 2, 8)-mesh embedded hypercube network. A wraparound connection is done along each row/column of the mesh if they have same label in Figure1 to deduce it to $(2,2,8)$-torus embedded hypercube network.

Scalability of a network [4], [5] is defined as the property by which the size of the system can be expanded with nominal changes in the existing configuration provided that system expansion results with improvement in performance. The torus embedded hypercube network is highly scalable network.

\section{COMPARISON OF RESULTS AND DISCUSSION}

For the analysis and comparison purpose we have considered network metrics such as Network Diameter and Topological Network cost. The diameter of a network can be defined as maximum number of hops an average message takes to reach to its destination [4], [9], [10].

The topological cost of a network depends on its node degree and diameter. A network with less node degree usually will have a large diameter, and a network with fewer diameters will possess larger node degree. Consequently, a network with large degree contains a large number of links while a network with low degree contains a small number of links [4].

\subsection{Network Diameter Analysis}

If the diameter is too large, it implies that a large number of nodes

Table 1. Comparison of Network Diameter

\begin{tabular}{|c|c|c|c|c|c|c|}
\hline Network type & 512 & 1024 & 2048 & 4096 & 8192 & 16384 \\
\hline $\begin{array}{l}(16,16, N)-\text { Mesh embedded } \\
\text { Hypercube }\end{array}$ & $\begin{array}{c}31 \\
N=2\end{array}$ & $\begin{array}{c}32 \\
N=4\end{array}$ & $\begin{array}{c}33 \\
N=8\end{array}$ & $\begin{array}{c}34 \\
N=16\end{array}$ & $\begin{array}{c}35 \\
N=32\end{array}$ & $\begin{array}{c}36 \\
N=64\end{array}$ \\
\hline $\begin{array}{l}\text { (1,m,16) - Mesh embedded } \\
\text { Hypercube }\end{array}$ & 14 & 18 & 26 & 34 & 48 & 66 \\
\hline $\begin{array}{l}(16,16, N)-T o r u s ~ e m b e d d e d \\
\text { Hypercube }\end{array}$ & $\begin{array}{c}17 \\
N=2\end{array}$ & $\begin{array}{c}18 \\
N=4\end{array}$ & $\begin{array}{c}19 \\
N=8\end{array}$ & $\begin{array}{c}20 \\
N=16\end{array}$ & $\begin{array}{c}21 \\
N=32\end{array}$ & $\begin{array}{c}22 \\
N=64\end{array}$ \\
\hline $\begin{array}{l}\text { (l,m,16) - Torus embedded } \\
\text { Hypercube }\end{array}$ & 10 & 12 & 16 & 20 & 26 & 36 \\
\hline
\end{tabular}

will have to be busy to get connected to the destination node. In other words, the message from source to destination will have to pass through larger number of intermediate nodes. This in turn brings down the performance of the whole system and hence system may slow down.

In the results given in Table 1 and Figure 2, as far as the network diameter is concerned, the torus embedded hypercube network needs lesser network diameter to get connected between a source node and a destination node. Hence the torus embedded hypercube network is much superior than mesh embedded hypercube network as far as performance metrics is concerned and much faster than an equivalent mesh embedded hypercube network.

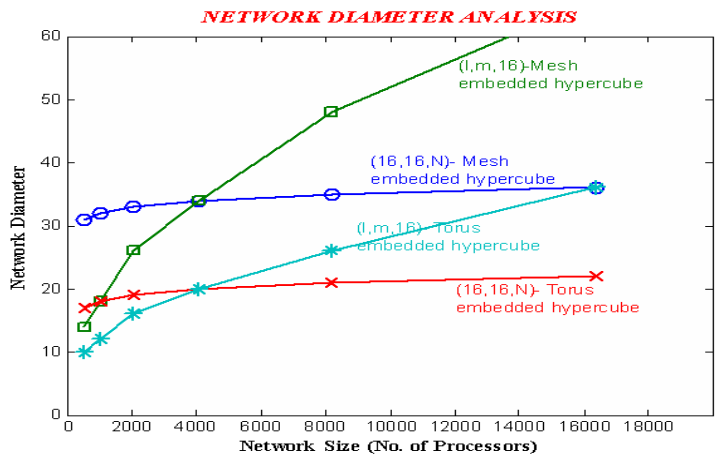

Figure 2. Network diameter analysis

\subsection{Network Cost Analysis}

The topological network cost analysis result is given in Table 2 and Figure 3.

Table 2. Comparison of Topological Network Cost

\begin{tabular}{|l|c|c|c|c|c|c|}
\hline Network type & $\mathbf{5 1 2}$ & $\mathbf{1 0 2 4}$ & $\mathbf{2 0 4 8}$ & $\mathbf{4 0 9 6}$ & $\mathbf{8 1 9 2}$ & $\mathbf{1 6 3 8 4}$ \\
\hline $\begin{array}{l}\text { (16,16,N)-Mesh embedded } \\
\text { Hypercube }\end{array}$ & $\begin{array}{r}155 \\
\mathrm{~N}=2\end{array}$ & $\begin{array}{c}192 \\
\mathrm{~N}=4\end{array}$ & $\begin{array}{c}231 \\
\mathrm{~N}=8\end{array}$ & $\begin{array}{c}272 \\
\mathrm{~N}=16\end{array}$ & $\begin{array}{c}315 \\
\mathrm{~N}=32\end{array}$ & $\begin{array}{c}360 \\
\mathrm{~N}=64\end{array}$ \\
\hline $\begin{array}{l}\text { (1,m,16)-Mesh embedded } \\
\text { Hypercube }\end{array}$ & 112 & 144 & 208 & 272 & 384 & 528 \\
\hline $\begin{array}{l}\text { (16,16,N)-Torus embedded } \\
\text { Hypercube }\end{array}$ & $\begin{array}{c}85 \\
\mathrm{~N}=2\end{array}$ & $\begin{array}{c}108 \\
\mathrm{~N}=4\end{array}$ & $\begin{array}{c}133 \\
\mathrm{~N}=8\end{array}$ & $\begin{array}{c}160 \\
\mathrm{~N}=16\end{array}$ & $\begin{array}{c}189 \\
\mathrm{~N}=32\end{array}$ & $\begin{array}{c}220 \\
\mathrm{~N}=64\end{array}$ \\
\hline $\begin{array}{l}\text { (1,m,16)- Torus embedded } \\
\text { Hypercube }\end{array}$ & 80 & 96 & 128 & 160 & 208 & 288 \\
\hline
\end{tabular}

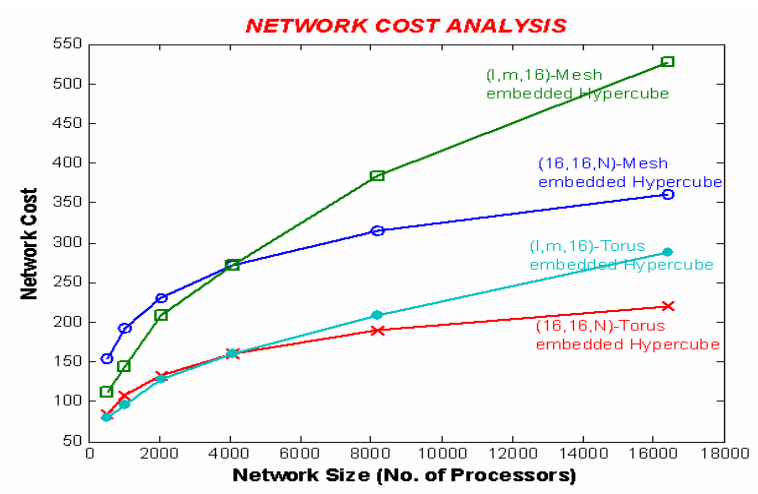

Figure 3. Network cost analysis 
Topology is the study of connectivity and continuity. It is seen that the torus embedded hypercube network will have low network cost. Though the network diameter is found to be increasing in $(1, \mathrm{~m}, 16)$-torus embedded hypercube network, it has to be noted that the torus embedded hypercube has better values for network cost as the system is scaled up.

\section{CONCLUSION}

We have analyzed a torus embedded hypercube interconnection network and compared its network parameters with mesh embedded hypercube interconnection network for a parallel architecture. Network metrics such as network diameter and topological network cost are considered since they are the most important parameters to justify the efficiency of the network.

It is necessary to come up with a network that is scalable, minimum network diameter and a minimum topological cost. All afore mentioned requirements are met by the torus embedded hypercube network and hence it can supersede the mesh embedded hypercube network. The results show that torus embedded hypercube network is much faster than the mesh embedded hypercube in terms of communication. Hence this network could be chosen as interconnection network for parallel architecture.

\section{APPENDIX}

To provide the basic principles, we have considered simple torus and hypercube networks with their data routing functions to show the connectivity among the nodes.

The data routing functions as in (1.a) to (1.d) of torus network [1] are

$$
\begin{aligned}
& T_{1}(i, j)=(i,(j+1) \bmod m) \\
& T_{2}(i, j)=(i,(m+j-1) \bmod m) \\
& T_{3}(i, j)=((i+1) \bmod l, j) \\
& T_{4}(i, j)=((l+i-1) \bmod l, j)
\end{aligned}
$$

where $i$ and $j$ are row and column numbers respectively.

According to these data routing functions, the various permutation cycles can be generated for a $2 \times 2$ torus network shown in Figure 4.

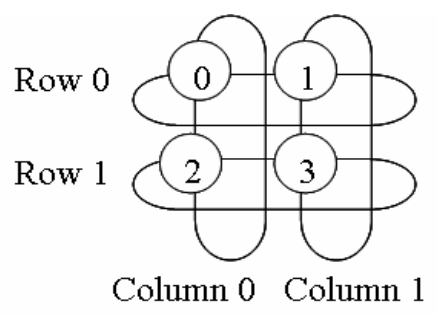

Figure 4. A $2 \times 2$ torus network

The data routing function as in (2.a) of hypercube network [1] is

$$
C_{d}\left(k_{21} \ldots k_{d+1} k_{d} k_{d-1} \ldots k_{0}\right)=\left(k_{k 1} \ldots k_{d+1} \bar{k}_{d} k_{d-1} . k_{0}\right)
$$

for $d=0,1, \ldots, n-1$ where $k_{j}$ for $(j=0$ to $n-1)$ is the binary representation of node address $\mathrm{k}$ and $\mathrm{n}=\log 2(\mathrm{~N})$ where $\mathrm{N}$ is the total number of nodes in the hypercube.

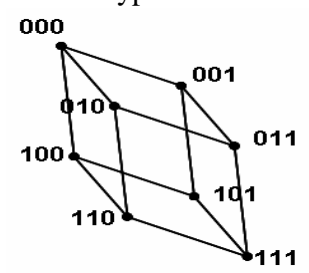

Figure 5. A 3-cube hypercube

According to the above hypercube data routing function the various permutation cycles can be generated for a 3-cube structured network shown in Figure 5.

\section{REFERENCES}

[1] K. Hwang and A. Briggs, "Computer Architecture and Parallel Processing," $1^{\text {st }}$ ed., McGraw-Hill, Inc. New York, 1990.

[2] Hesham El-Rewini and Mostafa Abd-El-Barr, " Advanced Computer Architecture and Parallel Processing," John Wiley \& Sons, Inc., Hoboken, New Jersey, 2005.

[3] J. L. Hennessy and D. A. Patterson, "Computer Architecture: A Quantitative approach," $3^{\text {rd }}$ ed., Morgan Kaufmann, 2005.

[4] Ahmed Louri and Hongki Sung, "An Optical Multi-Mesh Hypercube: A Scalable Optical interconnection Network for Massively Parallel Computing," Journal of Lightwave Technology, Vol. 12, No. 4, pp: 704-716, Apr. 1994.

[5] Ahmed Louri, "Optical Interconnection Networks for Scalable High-performance Parallel Computing Systems," Optical Interconnects Workshop for High Performance Computing, Oak Ridge, Tennessee, Nov 8-9, 1999.

[6] A. Ferna'ndez, K. Efe, "Product networks with logarithmic diameter and fixed degree," IEEE Transactions on Parallel and Distributed Systems, Vol. 6, No. 9, pp: 963-975, 1995.

[7] A. Ferna'ndez, "Homogeneous product networks for processor interconnection," PhD thesis, University of Southwestern Louisiana, Lafayette, October 1994.

[8] Zhaoyang Li, Yi Zhang, Yu Chen and Ruichun Tang, "Design and implementation of a high-performance interconnection network," Proceedings of the Fourth International Conference on Parallel and Distributed Computing, Applications and Technologies, PDCAT'2003, 27-29, Aug. 2003.

[9] N. Gopalakrishna Kini, M. Sathish Kumar and Mruthyunjaya H. S., "Analysis and Comparison of Torus Embedded Hypercube Scalable Interconnection Network for Parallel Architecture," International journal of Computer Science and Network Security, vol. 9, No.1, pp. 242-247, Jan. 2009.

[10] N. Gopalakrishna Kini, M. Sathish Kumar and Mruthyunjaya H.S., "A Torus Embedded Hypercube Scalable Interconnection Network for Parallel Architecture," IEEE explore conference publications, 2009. 